\title{
Experiência obtida em 100 transplantes de pâncreas
}

\section{Experience with 100 pancreas transplants}

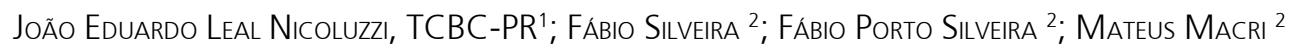

\section{RE S U M O}

\begin{abstract}
Objetivo: Relatar nossa experiência com 100 transplantes de pâncreas realizados em um período de sete anos. Métodos: Entre janeiro de 2001 e janeiro de 2008, 100 pacientes foram submetidos a transplante de pâncreas em nosso serviço, sendo 88 transplantes de pâncreas e rim simultâneo (TPRS) e 12 transplantes de pâncreas isolado (TPI). Todos foram transplantes primários. O manejo da porção exócrina do enxerto pancreático envolveu drenagem entérica em oito casos (todos TPRS) e a bexiga em 92 casos. O sistema venoso sistêmico do receptor foi utilizado para a drenagem venosa do enxerto em todos os casos. Nossos últimos 30 pacientes submetidos à TPRS não receberam terapia de indução independentemente do painel imunológico.Os pacientes TPRS receberam basiliximab e TPI receberam timoglobulina nos casos induzidos. Imunossupressão de manutenção foi realizada com tacrolimus, micofenolato mofetil e corticóides. O volume de perfusão do enxerto pancreático foi limitado a $800 \mathrm{ml}$ da solução de Celsior ou UW. Resultados: Demonstram que os enxertos ainda funcionantes são atualmente 64 dos 100 realizados. Perda do enxerto foi causada por: rejeição (oito pacientes), trombose venosa (nove pacientes), trombose arterial (um paciente) Complicações cirúrgicas encontradas: fístula anastomótica (tres pacientes), infecção peri-enxerto (10 pacientes), pancreatite do enxerto (cinco pacientes). A Rejeição foi observada com menos freqüência nos TPRS (5/92) que nos TPI (3/12). A morte ocorreu em 24 pacientes. Conclusão: Nossa impressão é que o transplante de pâncreas é altamente efetivo como terapia para o diabetes mellitus apesar da morbidade do procedimento.
\end{abstract}

Descritores: Transplante de pâncreas. Diabetes mellitus. Complicações cirúrgicas.

\section{INTRODUÇÃO}

A pós décadas de controvérsias em relação à eficácia terapêutica do transplante de pâncreas, o procedimento conquistou espaço e foi aceito como o tratamento preferencial para pacientes selecionados, com o diabetes mellitus do tipo I e complicações secundárias do diabetes, sobretudo as renais. É atualmente a única maneira de terapia de substituição endócrina em que seguramente se obtêm um estado euglicêmico e de normalidade da homeostasia glicêmica. Suas desvantagens incluem os riscos cirúrgicos do transplante de pâncreas e a necessidade de imunossupressão de maneira crônica'.

O Registro Internacional de Transplantes de Pâncreas, organizado em 1980, fornece dados históricos e atualizados do transplante de pâncreas. De 16 de dezembro de 1966 a dezembro de 2004, mais de 17000 transplantes de pâncreas foram realizados nos Estados Unidos, e aproximadamente 6000 em outros locais do mundo?

No Brasil, o transplante simultâneo de pâncreasrim tem ganhado aceitação desde a década de 90 , após legislação federal específica que garantiu a prioridade na alocação de órgãos para os candidatos a essa modalidade de transplante. O nosso programa de transplante de pâncreas teve inicio em janeiro de 2001.
Nosso objetivo é relatar nossa experiência com 100 transplantes de pâncreas realizados em um período de sete anos.

\section{MÉTODOS}

Entre janeiro de 2001 e janeiro de 2008, 100 pacientes foram submetidos ao transplante de pâncreas em nosso Serviço, sendo 88 deles transplante pâncreas-rim simultâneo (TPRS) e 12 transplantes de pâncreas isolado (TPI). Todos foram transplantes primários.

A duração do diabetes tende a ser menor em pacientes submetidos à TPI (média de $20 \pm 8$ anos) do que os TPRS ( $25 \pm 8$ anos). A incidência no sexo feminino foi proporcionalmente menor tanto no TPRS (43\%) quanto no TPI (47\%).

Em relação à técnica de drenagem da porção exócrina, utilizamos a drenagem entérica em oito pacientes (todos TPRS) e vesical nos outros 92 pacientes. O sistema de drenagem venosa foi o sistêmico em todos os transplantes.

Os enxertos pancreáticos foram considerados funcionantes pelo tempo que os receptores se mantivessem insulino-independentes. Falhas técnicas incluíram trom-

Trabalho realizado no Serviço de Transplante de Fígado e Pâncreas do Hospital Angelina Caron e Irmandade da Santa Casa de Misericórdia de Curitiba.

1. Doutor em Cirurgia. Cirurgião do Aparelho Digestivo; 2. Cirurgião do Aparelho Digestivo - Membro ABTO. 
bose do enxerto primária ou remoção de um enxerto funcionante por complicações como fístula, infecção perienxerto ou pancreatite. Outros casos foram considerados tecnicamente bem-sucedidos.

Em relação à imunossupressão, nossos últimos 30 pacientes submetidos à TPRS não receberam terapia de indução, independente do painel de anticorpos (PRA). Todos os outros receberam basiliximab no dia do transplante e no quarto dia pós-transplante. Todos os submetidos à TPI receberam timoglobulina $0,5 \mathrm{mg} / \mathrm{kg}$ por sete dias.

Todos os pacientes receberam tacrolimus oral (TAC) iniciado imediatamente no pós-transplante em uma dose de $0,1-0,2 \mathrm{mg} / \mathrm{kg}$ em duas doses diárias objetivando concentração sérica de 10-12ng/ml durante os três primeiros meses. Micofenolato mofetil (MMF) também foi iniciado imediatamente no pós-operatório na dose de 2-3g/dia em duas ou quatros doses diárias. Corticóides eram administrados da seguinte maneira: 500mg de metilprednisolona no intra-operatório, 250mg no primeiro pós-operatório e diminuído progressivamente até $30 \mathrm{mg} /$ dia aproximadamente entre o $7^{\circ}-10^{\circ}$ pós-operatório.

Todas as captações foram realizadas pela mesma equipe cirúrgica, treinada para a captação do enxerto de acordo com técnica padronizada, que preconiza a dissecção completa do enxerto à quente antes do clampeamento da aorta, sendo a perfusão realizada via aorta de maneira exclusiva. O volume da solução de preservação foi limitado a $800 \mathrm{ml}$ da solução de Celsior em nossos últimos 30 pacientes de TPRS, independente do peso do doador, com o objetivo de evitar pancreatite do enxerto por sobrecarga de volume. Todos os outros enxertos foram captados com a solução da Universidade de Wisconsin (UWBelzer) da mesma maneira. Os enxertos pancreáticos não foram reperfundidos ex-vivo, e foram armazenados a frio. Todos os doadores com menos de 55 ou mais de 10 anos de idade, sem histórico de diabetes mellitus, doença pancreática ou trauma pancreático eram considerados potenciais doadores. Hiperglicemia ou hiperamilasemia, instabilidade hemodinâmica necessitando drogas vasoativas (independente da dosagem) e períodos longos de internamento em terapia intensiva não eram considerados contra-indicações absolutas. A decisão final para a utilização do órgão era baseada no seu aspecto macroscópico, qualidade dos vasos sanguíneos, bem como as características macroscópicas após a perfusão.

As variáveis demográficas e de base são descritas de acordo com suas características estatísticas. Os dados em relação à modalidade do transplante são descritos pela frequência e porcentagem. Os dados contínuos são descritos pela média.

\section{RESULTADOS}

A idade do doador cadáver variou de 10 a 55 anos, sendo que os doadores tenderam a ser mais velhos no TPI que no TPRS. A média de idade para TPRS foi de $28 \pm 18$ anos e para TPI de 38 \pm 17 anos. O trauma foi a causa de óbito do doador em $69 \%$ dos mais jovens que 45 anos e $20 \%$ para os mais velhos que 45 anos. A incidência de doença cerebro-vascular foi a causa de óbito para o restante dos doadores.

O tempo de preservação variou pouco em relação à modalidade do transplante. Variou de 5-24 horas, sendo que o tempo médio foi de $11 \pm$ quatro horas. 0 tempo de preservação foi de 3-11, 12-23 e mais de 24 horas em $56 \%$, $42 \%$ e $2 \%$ dos casos, respectivamente.

Os resultados gerais demonstram que o número de enxertos pancreáticos funcionantes é de 64 após 100 transplantes realizados. Sendo que estratificando em diferentes períodos, 90\% dos doentes tiveram alta hospitalar com enxertos pancreáticos funcionantes. Com seis meses de seguimento ainda $85 \%$ dos doentes apresentavam enxertos funcionantes. Com um e cinco anos respectivamente 80 e $70 \%$, sendo que após este período outros seis perderam o enxerto por complicações adversas. As perdas de enxerto foram: rejeição (oito casos), trombose venosa (nove casos), trombose arterial (um caso), ou complicações cirúrgicas como fístula anastomótica (três casos), infecção peri-pancreática (cinco casos). A maior parte dos casos de pancreatite $(80 \%)$ ocorreu quando o tempo de preservação foi maior do que 18 horas. No gráfico 1 observa-se a evolução da experiência cirúrgica mostrando a maior parte das complicações nos primeiros anos do programa.

Os episódios de rejeição foram observados menos frequentemente na modalidade TPRS (cinco de $92 \mathrm{ca}-$ sos) do que em TPI (três de 12 casos). Para o diagnóstico de rejeição pancreática, a rejeição do rim foi utilizada como marcador e confirmada com biópsia pancreática. O uso da amilasúria foi decepcionante nesse sentido.

Óbito foi observado em 24 casos, sendo na maioria dos casos relacionadas a infecção (20 casos). Esses pacientes foram manejados por drenagem ampla e antibioticoterapia de largo espectro. Três desses casos foram manejados de maneira bem sucedida com transplantectomia. Observamos uma diminuição da incidência das taxas de infecção relacionadas a óbito quando paramos de realizar rotineiramente terapia de indução nos TPRS, sem observarmos aumento das taxas de rejeição. As quatro outras causas de morte foram acidente vascular cerebral hemorrágico, infarto agudo do miocárdio (dois casos) e acidente automobilístico.

\section{DISCUSSÃO}

Durante décadas a elevação dos níves séricos de glicose tem sido correlacionada com as complicações microvasculares do diabetes. Em decorrência das limitações da terapia insulínica de maneira intensiva, o transplante de células beta pancreáticas, através de um enxerto vascularizado (pâncreas), se mostrou efetivo e capaz de prolongar a sobrevida dos pacientes diabéticos tanto nefropatas quanto neuropatas ${ }^{1}$.

O sistema brasileiro de alocação de pâncreas para transplante é inicialmente estadual e depois em base naci- 


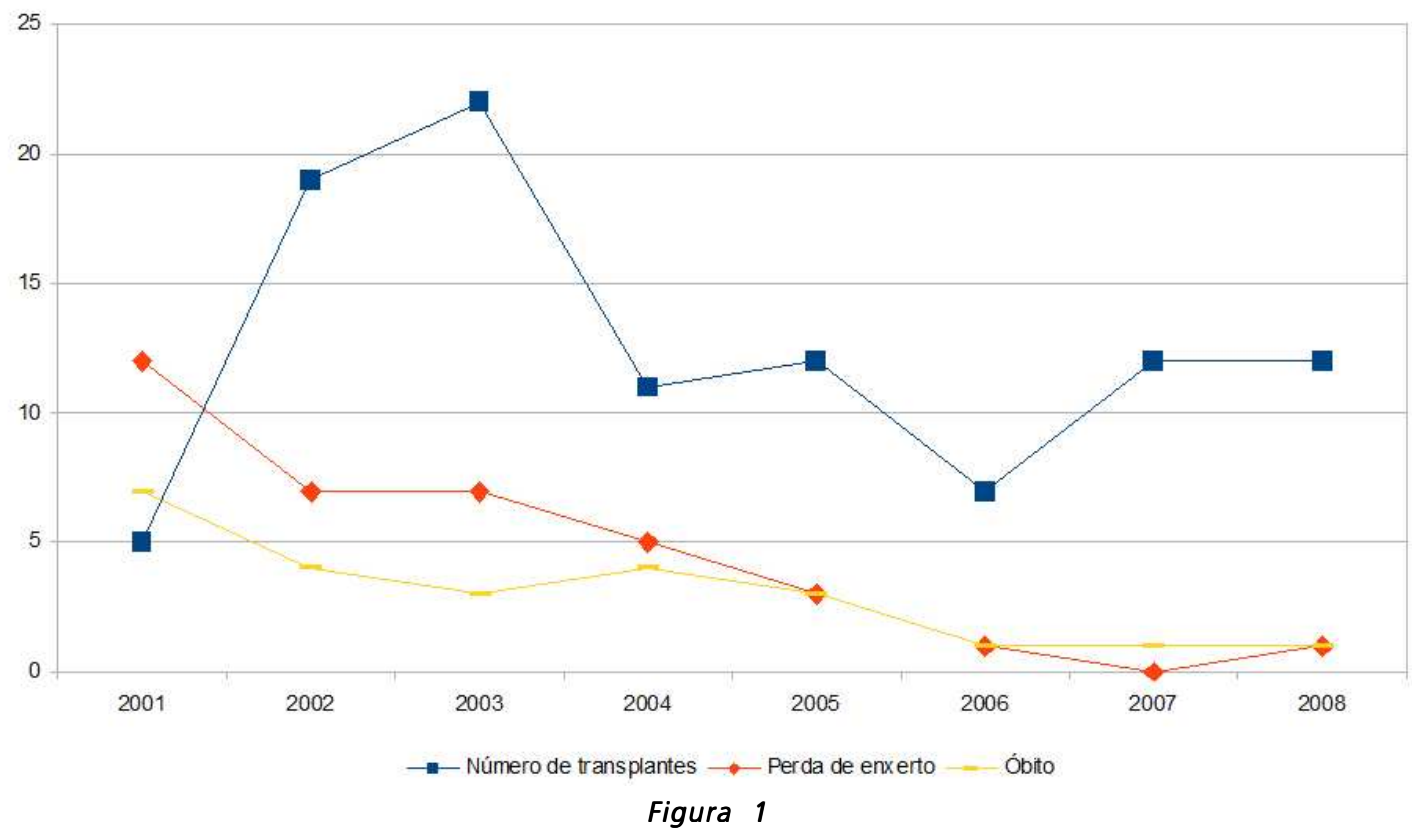

onal. Em nível estadual, a secretaria de saúde coordena as atividades de procura de órgãos. Os receptores são selecionados de acordo com a compatibilidade do sistema $A B O$ e teste de cross match negativo.

Nossos doadores preencheram os critérios de "doadores ideais", ou seja, vítima jovem, de trauma (causa externa) e sem comorbidades. Apesar dessas características demográficas, o uso de "doadores marginais" foi aceito, visto que as características macroscópicas do pâncreas durante a captação, antes e após a perfusão, era considerada a variável mais importante na determinação da adequabilidade do enxerto pancreático ${ }^{3}$. Essa abordagem, inspeção direta do enxerto, apesar de subjetiva, é adotada pela maioria dos especialistas ${ }^{4}$.

Nosso procedimento padrão de captação, quando possível, é firmemente baseado em dissecção a quente ampliada, com ligadura de todos os pequenos vasos sanguíneos e linfáticos peri-pancreáticos, atitude que claramente reduziu nossa incidência de sangramento pós-reperfusão, diminuiu tempo cirúrgico e intervenções pós-operatórias para tratamento de sangramentos e fístulas linfáticas.

Inicialmente nós utilizamos a solução da Universidade de Wiscosin (UW) para a preservação do pâncreas, utilizando baixo volume de solução $(800 \mathrm{ml})$ pela preocupação em relação ao edema pancreático e aumento da lesão de reperfusão. Durante o programa, o aumento da indisponibilidade no Brasil da solução UW, nos direcionou para o uso da solução de Celsior, que utilizando o mesmo volume $(800 \mathrm{ml})$, se provou tão eficaz quanto a solução UW para prevenir edema e pancreatite do enxerto pósreperfusão, assim como trombose do enxerto ${ }^{5,6}$.

Mais que em qualquer outro transplante de órgãos sólido, a discussão entre as diferentes técnicas cirúrgicas do transplante de pâncreas é controversa ${ }^{7}$. A drenagem venosa sistêmica associada à drenagem vesical das secreções exócrinas pancreáticas foi a técnica por nós escolhida. Mesmo considerando os conhecidos problemas de hiperinsulinemia periférica e complicações metabólicas e urológicas únicas ao método, essa técnica cirúrgica é segura e eficaz ${ }^{1}$. Atualmente, utilizamos a drenagem entérica-sistêmica de rotina, já que a utilização da amilasúria foi gradualmente sendo abandonada com o aumento da experiência no uso da tríade função renal - glicose sérica - biópsia renal, como marcador da rejeição. A drenagem vesical ainda é utilizada para pacientes de TPI.

Antes considerado um transplante de "alto-risco" em relação à imunossupressão, a alvorada de novos agentes após 1994 transformou o transplante de pâncreas. Desde o início de nosso programa em 2001, a terapia de indução com basiliximab (TPRS) ou timoglobulina (TPI) e manutenção com TAC-MMF-prednisona foi bem estabelecida na literatura ${ }^{8}$, e em consequência utilizada em nossos pacientes.

Apesar de existirem inúmeras razões para a utilização de terapia de indução, por exemplo, redução de rejeição precoce, acelerar função do aloenxerto renal e indução de efeito toleragênico ao aloantígneo do doador, uma importante modificação de nosso protocolo ocorreu quando suspendemos o uso da terapia de indução anticélulas T para o TPRS, estratégia utilizada em outros centros $^{8}$, sendo que nosso objetivo era a redução das complicações infecciosas locais ${ }^{9}$. A retirada do esteróide, um ano após o transplante, em pacientes sem episódios de rejeição, será o nosso próximo objetivo.

Nós observamos uma redução das complicações cirúrgicas ano após ano, experiência também relatada em outras séries ${ }^{1,10}$. A Trombose venosa foi uma grande causa de perda de enxerto em nossa série. A incidência dessa complicação foi reduzida de maneira marcante após 2003, após iniciarmos a realização de rotina da liberação completa de todos os vasos ilíacos. Apenas dois pacientes per- 
deram seus enxertos devido a essa complicação após 2003. Não observamos que a heparinização reduziu o risco de trombose, entretanto isso não é universalmente observado ${ }^{11,12}$.

A incidência de complicações urológicas da drenagem vesical é uma constante, e não reduziu com o passar do tempo, assim como observado por outros ${ }^{13,14}$, mas a gravidade raramente foi extrema. A incidência de outras complicações cirúrgicas (trombose arterial, fístula duodenal, pancreatite) se mostrou pontual e semelhante à da literatura ${ }^{15,16}$.
Nossa impressão, apesar de complicações cirúrgicas e de imunossupressão, é que o transplante de pâncreas é uma terapia altamente eficaz para o diabetes mellitus, e após sete anos de programa e cem transplantes realizados, concluímos que ele tem papel definido no manejo deste frágil e seleto grupo de doentes.

\section{Agradecimentos}

O autor agradece a todas as pessoas que sabidamente estiveram envolvidas durante a empreitada descrita no presente artigo.

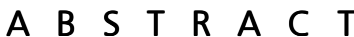

Objective: Report our experience with 100 pancreas transplants performed in a period of seven years. Methods. Between January 2001 and January 2008, 100 patients underwent pancreatic transplantation at our center, 88 simultaneous pancreas kidney transplantation (SPK) and 12 pancreas transplantation alone (PTA). All of these were primary transplants. Pancreas graft management of the exocrine drainage technique involved enteric drainage in 8 (all SPK) and bladder in 92 cases. The recipient systemic venous system was used for the pancreas graft venous effluent in all cases. Our last thirty patients submitted to SPK did not receive any induction therapy regardless of the PRA. SPK received basiliximab and PTA patients received thymoglubulin. Maintance imunossupression was with TAC, MMF and corticosteroids. Graft perfusion volume was limited to $800 \mathrm{ml}$ of Celsior or UW solution. Results. Overall results show that the number of functioning pancreatic grafts is 64 after 100 performed. Graft losses were: rejection (8 cases), venous thrombosis (9 cases) arterial thrombosis(1 case), or surgical complications such as anastomotic leak (3 cases), perigraft infection (10 cases), pancreatitis of the graft(5 cases). Rejection was observed less frequently in SPK recipients 5 cases (5/92 recipients) than PTA recipients (3/12). Death was observed in 24 cases. Conclusion. Our impression is that pancreas transplantation is highly effective therapy for diabetes mellitus and there is still a role in the diabetes treatment for allograft transplantation in a near future.

Key words: Pancreas transplantation. Diabetes mellitus. Surgical complications.

\section{REFERÊNCIAS}

1. Sutherland DE, Gruessner RW, Dunn DL, Matas AJ, Humar A, Kandaswamy $R$ et al. Lessons learned from more than 1,000 pancreas transplants at a single institution. Ann Surg. 2001; 233(4):463-501.

2. Gruessner AC, Sutherland DE. Pancreas transplant outcomes for United States (US) and non-US cases as reported to the United Network for Organ Sharing (UNOS) and the International Pancreas Transplant Registry (IPTR) as of June 2004.Clin Transplant. 2005; 19(4):433-55.

3. Iwanaga Y, Sutherland DE, Harmon JV, Papas KK. Pancreas preservation for pancreas and islet transplantation. Curr Opin Organ Transplant. 2008; 13(2):135-41.

4. Gruessner RWG. Donor procedures. Gruessner RWG, Sutherland DER, editors. Transplantation of the pancreas. New York: SpringerVerlag; 2004. p. 126-42.

5. Boggi U, Vistoli F, Del Chiaro M, Signori S, Croce C, Pietrabissa A et al. Pancreas preservation with University of Wisconsin and Celsior solutions: a single-center, prospective, randomized pilot study. Transplantation. 2004; 77(8):1186-90.

6. Nicoluzzi J, Macri M, Fukushima J, Pereira A. Celsior versus Wisconsin solution in pancreas transplantation. Transplant Proc. 2008; 40(10):3305-7.

7. Stratta RJ, Gaber AO, Shokouh-Amiri MH, Reddy KS, Alloway RR, Egidi MF et al. Evolution in pancreas transplantation techniques: simultaneous kidney-pancreas transplantation using portal-enteric drainage without antilymphocyte induction. Ann Surg. 1999; 229(5):701-8; discussion 709-12.

8. Singh RP, Stratta RJ. Advances in immunosuppression for pancreas transplantation. Curr Opin Organ Transplant. 2008; 13(1):79-84.

9. Nicoluzzi J. Successful pancreas-renal transplantation without induction therapy. Transplant Proc. 2005; 37(10):4438-9.

10. Rossi M, Lai Q, Spoletini G, Poli L, Nudo F, Ferretti S, et al. Simultaneous pancreas-kidney transplantation: a single-center experience and prospective analysis. Transplant Proc. 2008; 40(6):2024-6.

11. Humar A, Kandaswamy R, Granger DK, Gruessner RW, Gruessner $A C$, Sutherland DE. Decreased surgical risks of pancreas transplantation in the modern era. Ann Surg. 2000; 231(2):269-75.

12. Sollinger $H$. Pancreatic transplantation and vascular graft thrombosis [editorial]. J Am Coll Surg. 1996; 182(4):362-3.

13. Sindhi R, Stratta RJ, Lowell JA, Sudan D, Cushing KA, Castaldo $P$, Jerius JT. Experience with enteric conversion after pancreatic transplantation with bladder drainage. J Am Coll Surg. 1997; 184(3):281-9.

14. Ploeg RJ, Eckhoff DE, D'Alessandro AM, Stegall MD, Knechtle SJ, Pirsch JD et al. Urological complications and enteric conversion after pancreas transplantation with bladder drainage. Transplant Proc. 1994; 26(2):458-9.

15. Reddy KS, Stratta RJ, Shokouh-Amiri MH, Alloway R, Egidi MF, Gaber AO. Surgical complications after pancreas transplantation with portal-enteric drainage. J Am Coll Surg. 1999; 189(3):305-13.

16. Pirsch JD, Odorico JS, D'Alessandro AM, Knechtle SJ, Becker BN, Sollinger HW. Posttransplant infection in enteric versus bladderdrained simultaneous pancreas-kidney transplant recipients. Transplantation. 1998; 66(12):1746-50.

Recebido em 05/02/2009

Aceito para publicação em 07/04/2009

Conflito de interesse: nenhum

Fonte de financiamento: nenhuma

\section{Como citar esse artigo:}

Nicoluzzi JEL, Silveira F, Silveira FP. Experiência obtida em 100 transplantes de pâncreas. Rev Col Bras Cir. [periódico na Internet] 2010; 37(2). Disponível em URL: http://www.scielo.br/rcbc

\section{Endereço para correspondência:}

Dr João Eduardo Nicoluzzi

E-mail: jenicoluzz@yahoo.com 